\title{
The Effect of Motion on the Perception of Material Appearance
}

\author{
Ruiquan Mao \\ Universidad de Zaragoza, I3A \\ maohuskar@unizar.es \\ Belen Masia \\ Universidad de Zaragoza, I3A \\ bmasia@unizar.es
}

\author{
Manuel Lagunas \\ Universidad de Zaragoza, I3A \\ mlagunas@unizar.es \\ Diego Gutierrez \\ Universidad de Zaragoza, I3A \\ diegog@unizar.es
}

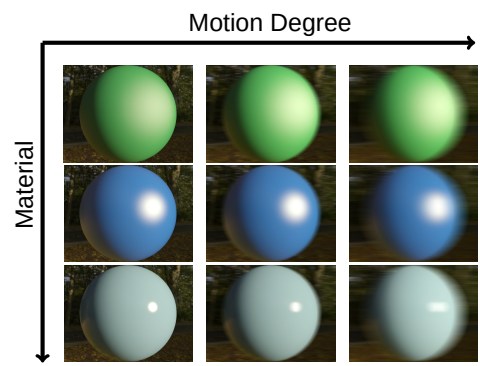

(a)

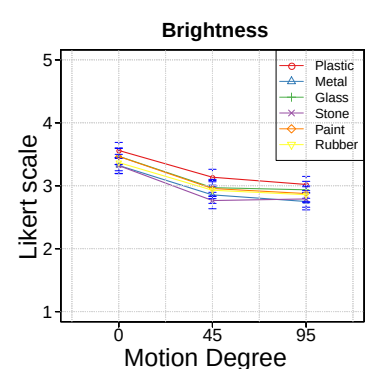

(b)

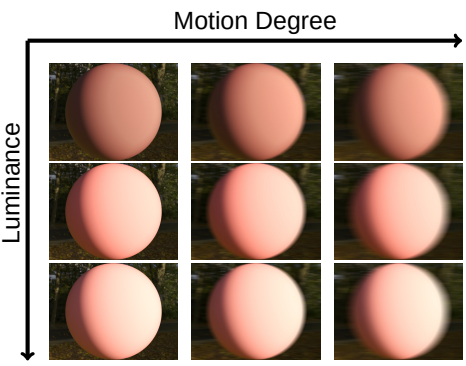

(c)

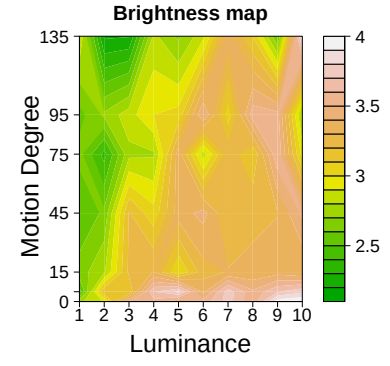

(d)

Figure 1: We conduct two large-scale crowdsourced experiments to analyze the influence of motion blur in the perception of material appearance. (a) Representative samples of the stimuli used in our first experiment, in which we analyze the perception of a series of high-level attributes for varying degrees of motion and different materials. (b) The variation of the high-level attribute brightness for different motion degrees and material categories tested in our first experiment. (c) Representative samples of the stimuli used in our second experiment, in which we look at the influence of varying luminance levels and motion degree on perceived brightness. (d) Results of our second experiment, showing a non-linear influence of motion degree and luminance level on perceived brightness.

\begin{abstract}
We analyze the effect of motion in the perception of material appearance. First, we create a set of stimuli containing 72 realistic materials, rendered with varying degrees of linear motion blur. Then we launch a large-scale study on Mechanical Turk to rate a given set of perceptual attributes, such as brightness, roughness, or the perceived strength of reflections. Our statistical analysis shows that certain attributes undergo a significant change, varying appearance perception under motion. In addition, we further investigate the perception of brightness, for the particular cases of rubber and plastic materials. We create new stimuli, with ten different luminance levels and seven motion degrees. We launch a new user study to retrieve their perceived brightness. From the users' judgements, we build two-dimensional maps showing how perceived brightness varies as a function of the luminance and motion of the material.
\end{abstract}

\section{KEYWORDS}

Material Perception, Dynamic Environment, Material Definition Language (MDL), Motion blur

\section{INTRODUCTION}

The perception of material appearance is affected by confounding effects such as the shape of the object, illumination, viewing conditions, etc. However, being able to distinguish between materials and to infer their key properties by sight is an inherent process in humans, which is invaluable for multiple tasks. How this is done exactly remains unclear, since human perception is a complex process influenced by a large set of variables. In fact, a unified theory that fully explains such a process does not exist [Anderson 2011; Fleming 2014].

To reduce the dimensionality of the perception of material appearance, many works have focused on developing applications for material synthesis [Zsolnai-Fehér et al. 2018], editing [Serrano et al. 2016], or filtering [Jarabo et al. 2014]. Other works focus on investigating individual variables that may affect our perception, 
such as translucency [Gkioulekas et al. 2015], or gloss [Pellacini et al. 2000; Wills et al. 2009].

With a few exceptions, most of the research on material perception has focused on static stimuli. Doerschner et al [2011] identified three motion cues the brain could rely on to distinguish between matte and shiny surfaces. Aliaga et al. [2015a] explore the interplay of cloth and dynamics, while Sakano et al. [2008] studied how self-motion influences the perception of gloss. Nevertheless, a generalized approach that tries to explain the effect of motion on a wide variety of attributes remains an open avenue of research.

In this paper, we study the influence of linear motion in the perception of material appearance relying on a set of intuitive, high-level perceptual attributes [Serrano et al. 2016]. Inspired by previous large-scale studies [Rubinstein et al. 2010; Serrano et al. 2016], we rely on crowdsourced data, from a set of 72 varied, realistic materials. To create a dense space of stimuli, we add additional variations by modifying their luminance, and render them with different levels of motion. This creates a total of 284 stimuli, used in two different experiments.

In the first experiment, we statistically analyze the significant change in how several of these high-level attributes are perceived according to the degree of motion. While brightness decreases significantly, more diffuse appearances remain constant as motion increases. This may be explained by the fact that motion blur has a higher impact on the high frequencies of an image which are usually associated with more specular materials.

In a second experiment, we focus on the perception of brightness, where the first experiment showed a larger influence of motion. We select rubber and plastic materials, with seven motion degrees and ten luminance levels. Using human judgements through Mechanical Turk, we build a brightness map as a two-dimensional function that shows the average perceived brightness for each level of luminance and degree of motion.

Our contributions represent just a step towards a better understanding of how motion affects material perception, a relatively unexplored topic compared to studies on static stimuli. To encourage further research on this topic, all our data will be made publicly available, including the material database, code, and the users' responses.

\section{RELATED WORK}

\subsection{Material perception}

To understand the perceptual properties of a given material is a complex task given the ambiguity of the information that arrives at our visual system, influenced by illumination, shape, motion, and other confounding factors. Nevertheless, humans show an outstanding performance recognizing materials [Fleming 2014; Fleming and Bülthoff 2005; Ged et al. 2010; Li and Fritz 2012]. We are able to infer the physical properties of objects without the need to touch them [Fleming et al. 2015a, 2013; Maloney and Brainard 2010; Nagai et al. 2015; ?], just by briefly looking at them [Sharan et al. 2009, 2008]. To quantify the contribution of each visual and non-visual cue in our perception remains an open venue of research [Anderson 2011; Fleming 2014; Fleming et al. 2015b; Maloney and Brainard 2010]. There exist some evidence that illumination [Fleming et al. 2003; Křivánek et al. 2010], shape [Havran et al. 2016; Vangorp et al. 2007] or motion [Doerschner et al. 2011; Hartung and Kersten 2002] may be one of these factors that alter our perception of a given material. Our work is similar to the latter as we focus on the effect of motion in our perception of material appearance. We launch two experiments using high-level explainable attributes and several stimuli with different degrees of motion and luminance. Then, we analyze the disparity in users' answers as the degree of movement in the stimuli varies and construct brightness maps from participants' responses.

\subsection{Dynamic environment}

The effect of motion in perception has been an active area of research [NEFS 2008; Ullman 1979]. Sakano [2008] study the effect of self-motion in our perception of glossiness. Later extended to describe reflectance properties of a given material [Doerschner et al. 2011] - where they found three visual cues related to motion that our brain could rely on to describe to distinguish matte or shiny surfaces -, and to modulate material appearance [Marlow and Anderson 2016]. Navarro et al. [2011] link perceptual attributes to low-level scene parameters, like the shutter speed of a virtual camera or the anti-aliasing in a scene, for motion blur rendering. The effect of motion has been also studied on the perception we have about liquids [Kawabe et al. 2015], cloth [Aliaga et al. 2015a], or depth [Braunstein 2014]. To see if the responses of our visual system change under motion [Kam et al. 2015], or to improve our perception of glossiness [Tani et al. 2013]. Unlike previous work, we do not focus on a particular property or application; instead, we follow a general approach in order to take a step towards the understanding of the effect of motion in a set of high-level attributes that can describe a wide variety of materials.

\subsection{Material modeling}

In order to faithfully represent the real-world, we need models that capture its richness, are flexible, and can be used easily. There have been many efforts to measure materials and represent them in a digital form. MERL [Matusik et al. 2003] dataset has a set of 100 isotropic materials measured from real samples, UTIA [Filip and Vávra 2014] also adds anisotropy to its set of materials. Objects under Natural Illumination Database [Lombardi and Nishino 2012] includes calibrated HDR information, and the recent database by Dupuy and Jakob [2018] also adds spectral reflectances to its samples. However, measured models usually lack flexibility and are expensive to evaluate, therefore, in our work we decided to work with the database $v$ Materials ${ }^{1}$, a curated collection of realistic materials and lights represented in MDL (Material Definition Language) [Kettner et al. 2015] that can be rendered with the OptiX ray tracing engine [Parker et al. 2010]. The ability to model realistic materials together with the studies on material perception has encouraged the development of perceptual applications. Nielsen et al. [2015] present a novel mapping of measured BRDFs, allowing for the extraction of descriptive principal components later used by Serrano et al. [2016] to find a relationship between perceptual attributes and an underlying PCA-based representation of BRDFs. To find a metric that models the relationship between human judgements is a long-standing problem not solved yet [Garces et al. 2014; Lagunas

\footnotetext{
${ }^{1}$ https://www.nvidia.com/en-us/design-visualization/technologies/vmaterials/
} 
et al. 2018; Soler et al. 2018; Wills et al. 2009; ?]. Lagunas et al. [2019] derive a metric on image space, based on deep learning models, that correlates with human perception and improves previous baselines [Ngan et al. 2006; Pereira and Rusinkiewicz 2012; Sun et al. 2017]. These metrics can also be computed directly over measured BRDFs [Fores et al. 2012; Ngan et al. 2005], or on perceptual traits [Pellacini et al. 2000; Serrano et al. 2016]. Our work diverges from the latter since it does not aim to build a perceptual application, instead, we focus on the more fundamental problem of evaluating the differences in human perception of material appearance under the influence of motion.

\section{STIMULI CREATION}

The following section explains the process followed in order to create the stimuli for our experiments.

Materials. . We use the material library $v$ Materials, a large collection of realistic materials and lights described in Material Definition Language (MDL) [Kettner et al. 2015]. We did not use a measured material database since they will not give us the efficiency needed to, in the future, use high-level material features to control and edit material appearance in real-time. Among all the available options, we selected a subset of 72 materials that span six categories, including glass, metal, paint, plastic, rubber, and stone. We decided to choose materials categories that represent daily items to avoid introducing bias due to unawareness in the participants' answers.

The materials in vMaterials include additional layers that add texture or small geometric details at rendering time. These features can alter the information that arrives at our visual system and distract the participant from its real purpose which is to observe the material itself. Therefore, in order to focus the attention of the user, we decided to remove all additional layers included in the material description, obtaining at the end, a homogeneous representation of the material. This procedure is depicted in Figure 2.

Adding motion. To approximate the effect of motion in our stimuli, we use an image algorithm consisting in a two-dimensional filter [Bradski 2000], described in Equation 1.

$$
\mathcal{I}_{o}(x, y)=\sum k\left(x^{\prime}, y^{\prime}\right) \times \mathcal{I}_{i}\left(x+x^{\prime}, y+y^{\prime}\right)
$$

where $\mathcal{I}_{i}(x, y)$ and $\mathcal{I}_{o}(x, y)$ are the input and output images respectively; and $(x, y)$ are the coordinate of the pixel in the image. The expression $k\left(x^{\prime}, y^{\prime}\right)$ means a convolution kernel, whose index in the kernel is $\left(x^{\prime}, y^{\prime}\right)$. The range of $k\left(x^{\prime}, y^{\prime}\right)$ is $1 \leq x^{\prime} \leq k_{c}$ and $1 \leq y^{\prime} \leq k_{r}$, where $k_{c}$ and $k_{r}$ are the number of columns and rows in $k$. When the aperture is partially outside the image, we interpolate outlier pixel values by mirroring $\mathcal{I}_{i}$.

Our experiments only intend to investigate material perception for linear movement, therefore $k\left(x^{\prime}, y^{\prime}\right)$ is a horizontal vector, whose value is $1 / k_{c}$. The size of the kernel $\left(k_{c}\right)$, can be considered as the motion degree. In a static situation, the motion degree is 0 .

Scene. . We use a sphere as the 3D model, a well-known surface, widely used in previous user-studies [Fleming et al. 2003; Jarabo et al. 2014]. The 3D model is placed in the centre of the scene. In order to render our stimuli, first, we use OptiX [Parker et al. 2010], a general purpose ray tracing engine, with the homogeneous material definition to generate an image of a static material, then we apply

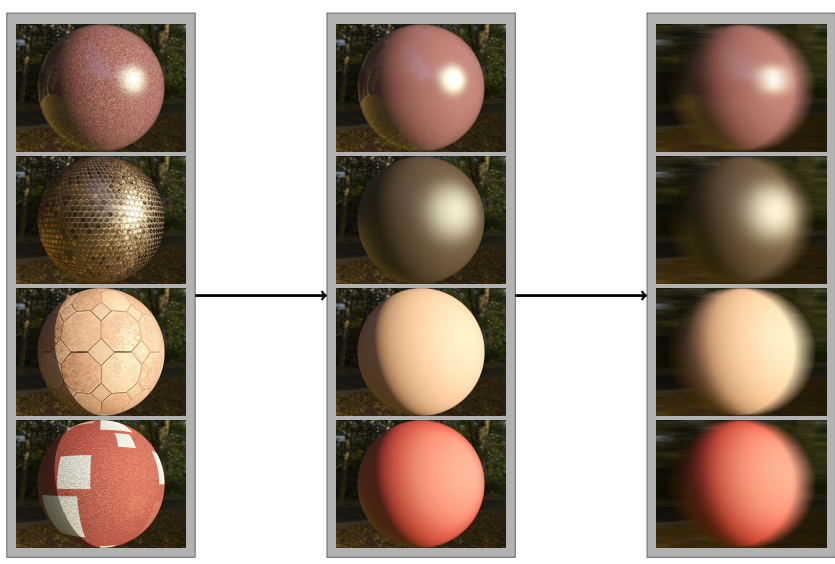

Figure 2: Summary of the process followed to generate stimuli of homogeneous materials with different degrees of motion. From left to right, the first column shows the frameworks used for rendering. The second column is the rendering result using $\boldsymbol{v}$ Materials and ray tracing engine OptiX [Parker et al. 2010], including texture and small geometric details. The third column represents the homogeneous material removing additional information provided in vMaterials. The last column shows the stimuli with different degrees of motion.

the two-dimensional filter explained in Equation 1 to add motion to the scene. Figure 2 summarizes the process of stimuli creation.

\section{EXPERIMENT 1: RATING MATERIAL ATTRIBUTES}

This section describes the approach followed in order to collect human ratings of perceptual attributes on materials under the influence of a certain degree motion.

Stimuli. We choose a set of 72 different realistic materials, that span six different material categories, and three degrees of motion: 0,45 , and 95 with a significant difference between them in order to provide a notable change in the stimuli appearance. Further explanation about the stimuli creation is given in Section 3. A subset of the stimuli is shown in Figure 3, all the stimuli are shown in the supplementary material.

Participants. Similar to previous work dealing with large-scale experiments in computer graphics [Lagunas et al. 2019; Rubinstein et al. 2010; Serrano et al. 2016], we rely on Amazon Mechanical Turk (MTurk) to launch our experiments. In the end, a total of 540 users performed the task. Users were unaware of the purpose of the experiment.

Procedure. Our user-study deals with the perception of material appearance under the influence of motion. We ask the users to rate a set of perceptual attributes regarding the material in the stimuli. They rate the attributes in a Likert-like scale, which has proven to work reliably in multi-modal problems [Yumer et al. 2015]. Following previous work [Du et al. 2013; Serrano et al. 2016; Zell et al. 2015], our scale ranges from 0 (very little) to 5 (a lot). We 


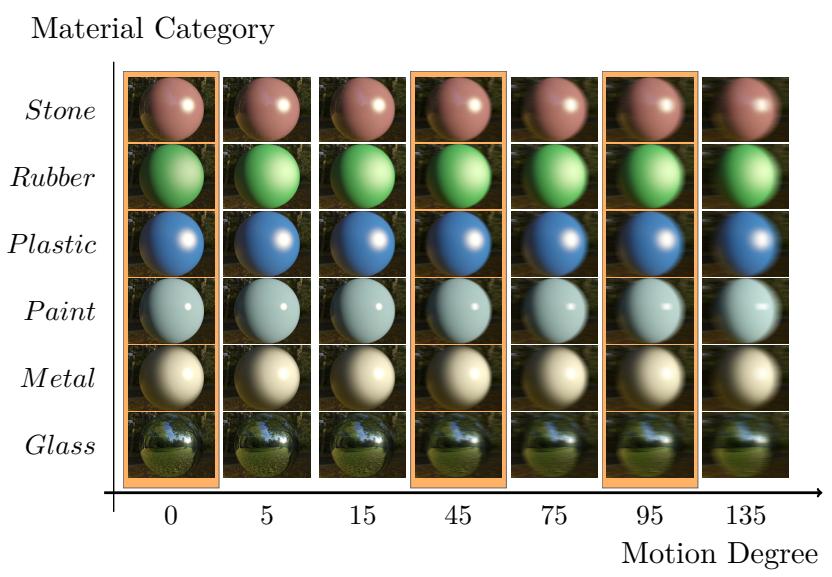

Figure 3: Subset of the stimuli used in the user experiment. Each row correspond to a material from a material category. The $x$-axis shows the degree of motion, going from 0 (a static stimuli) to 135 (fastest stimuli). For the first experiment, we only use the motion degrees 0,45 , and 95 since they provide significant visual changes in the final renderings.

consider this range a good compromise between the complexity to fill in the survey and the number of available options for the participants.

Following the work of Serrano et al. [2016], participants rate 14 perceptual attributes, namely: plastic-like, rubber-like, metalliclike, fabric-like, ceramic-like, soft, hard, matte, glossiness, brightness, roughness, tint of reflections, strength of reflections, and sharpness of reflections. Covering both, high and mid-level features of the material, allowing for a comprehensive evaluation of the participants' perception.

Each test consisted of 12 different trials, 4 groups of three spheres with the same material and different motion degrees, randomly displayed. The participants had to rate all the 14 perceptual attributes in all the trials of the user study (HIT, Human Intelligence Tasks, in MTurk terminology). The experiment was developed to work in standard web browsers. Before the real experiment, there is a thorough description of the task, and a brief training session in order to minimize worker unreliability [Welinder et al. 2010]. During the real test, participants were shown one stimulus at a time. In the end, each stimulus was rated by 30 different users and we collected a total of 6480 answers for 14 attributes, yielding 90.720 ratings.

\subsection{Analysis}

Among the 14 perceptual attributes rated by the participants, we select a subset of six to perform the statistical analysis, those include brightness, glossiness, matte, roughness, sharpness of reflections and strength of reflections. We conducted a Friedman rank sums test, a non-parametric version of ANOVA,[Pohlert 2018] in order to analyze how the factors: material category and motion degree have affected participants' answers. The motion degree contains three levels: 0,45 , and 95 while the material category has six: glass, metal, paint, plastic, rubber, and stone. We decided to use a Friedman test since it is suitable for Likert-like ratings and samples do not need to be normally distributed. We chose a significance level of $p=0.05$ in all our tests.

The influence of motion. A summary with all the $p$-values for each rated attribute and material category can be found in Table 1, a complete table with all the $p$-values can be found in the supplementary material. We observe that glossiness, brightness, strength of reflections, and sharpness of reflections have a significant change in all the material categories $(p<0.05)$. On the other hand, the attributes matte, and roughness are not influenced by motion and do not have a significant change for all the material categories $(p>0.05)$. These results may suggest that motion has a bigger effect on our perception of the attributes that describe the specularity of the material instead of on the ones that are used to characterize diffuse appearances.

The influence of the material. The attributes matte, and roughness are not influenced by motion in all the material categories. Only metal and paint materials for the matte attribute will have a significant change $(p<0.05)$. We argue that this significant change is produced because these materials have a characteristic narrow specular lobe that under the effect of motion blur will broaden, giving them a more diffuse and dimmer final appearance.

Given the previous findings, we further investigate the significance of each factor individually. We conduct a Nemenyi post hoc test with a single-step $p$-value adjustment, suitable to find statistically significant groups after the Friedman test. Table 2 shows the $p$-values for the attributes glossines, brightness, strength of reflections, and sharpness of reflections; and both factors: material category, and motion degree. We can observe a clear trend for all four attributes, there is a significant change in participants' perception when the stimuli changes from static to a middle motion degree $(p<0.05)$ and if the stimulus moves from static to the highest analyzed motion degree $(p<0.05)$. However, if the stimulus changes from a middle motion degree (45) to the highest motion degree (95), there are no significant changes in participants' answers $(p>0.05)$. This showcases the non-linear nature of human perception of material appearance [Thompson et al. 2011] and tells us that linear changes in motion do not necessarily correlate with linear changes on the perception we have about the stimuli [Champion and Warren 2017; Zanker 1995]. However, the rubber material category does not show significant changes for attributes glossiness, and strength of reflections when the degree of motion changes from static to a middle motion degree $(p>0.05)$, but shows significant changes when we move from a static to the highest motion degree $(p<0.05)$. This could be due to the diffuse appearance of rubber, which lacks reflections. Therefore, small changes in motion do not produce perceptually visible changes in the specular appearance of the material, requiring higher motion degrees to perceive those changes.

Figure 4 shows the trends of participants' ratings for each of the six perceptual properties and motion degrees. As previously discussed glossines, brightness, sharpness of reflections and strength of reflections attributes have significantly different answers under motion. For each attribute, we observe a falloff as the motion degree is increased. This is expected as the blur introduced by the movement of the stimulus dims the image content, soften highlights. Also, if we observe the sharpness of reflections attribute, we see 
Table 1: Results from Friedman rank test for all the materials and attributes over all motion degrees. The last two columns show the results from the Friedman test $\left(\chi^{2}\right.$ and p-values).

\begin{tabular}{|c|c|c|c|}
\hline Attribute & Material & $\chi^{2}$ & $p$-value \\
\hline \multirow{6}{*}{ 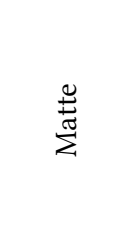 } & Plastic & 0.301 & 0.860 \\
\hline & Metal & 13.942 & 0.001 \\
\hline & Glass & 0.833 & 0.659 \\
\hline & Stone & 1.240 & 0.538 \\
\hline & Paint & 10.033 & 0.007 \\
\hline & Rubber & 2.898 & 0.235 \\
\hline \multirow{6}{*}{ 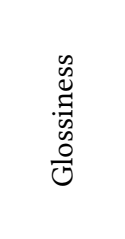 } & Plastic & 24.958 & 0.004 \\
\hline & Metal & 19.080 & 0.072 \\
\hline & Glass & 52.166 & 0 \\
\hline & Stone & 64.045 & 0 \\
\hline & Paint & 29.110 & 0 \\
\hline & Rubber & 19.648 & 0.054 \\
\hline \multirow{6}{*}{ 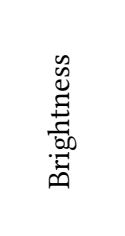 } & Plastic & 70.490 & 0 \\
\hline & Metal & 52.211 & 0 \\
\hline & Glass & 62.027 & 0 \\
\hline & Stone & 72.240 & 0 \\
\hline & Paint & 71.274 & 0 \\
\hline & Rubber & 59.049 & 0 \\
\hline \multirow{6}{*}{ 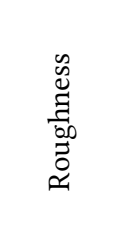 } & Plastic & 2.924 & 0.232 \\
\hline & Metal & 1.769 & 0.413 \\
\hline & Glass & 2.233 & 0.327 \\
\hline & Stone & 1.347 & 0.510 \\
\hline & Paint & 1.327 & 0.515 \\
\hline & Rubber & 0.003 & 0.999 \\
\hline \multirow{6}{*}{ 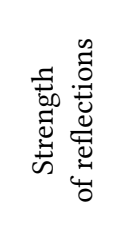 } & Plastic & 41.848 & 0 \\
\hline & Metal & 42.993 & 0 \\
\hline & Glass & 66.824 & 0 \\
\hline & Stone & 29.444 & 0 \\
\hline & Paint & 18.885 & 0.079 \\
\hline & Rubber & 14.236 & 0.001 \\
\hline \multirow{6}{*}{ 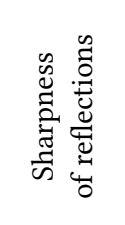 } & Plastic & 45.868 & 0 \\
\hline & Metal & 51.283 & 0 \\
\hline & Glass & 101.800 & 0 \\
\hline & Stone & 48.995 & 0 \\
\hline & Paint & 38.979 & 0 \\
\hline & Rubber & 34.243 & 0 \\
\hline
\end{tabular}

that material categories with clear reflections in their materials like glass - have higher ratings in the static stimulus and a steeper decreasing slope. On the other hand, matte and roughness attributes, not considered statistically significant, have slight changes as we increase motion, showing an almost uniform behavior.

\section{EXPERIMENT 2: BRIGHTNESS MAP CONSTRUCTION}

Given the results of the previous experiment (Section 4), where we found that the attributes that describe the specularity of material
Table 2: Results from the Nemenyi post hoc test. The first column is the name of the attribute rated by the participants. The names of the material categories are placed in the second column. From the third to the fifth column we have the p-values for each change in motion degree.

\begin{tabular}{|c|c|c|c|c|}
\hline \multirow{2}{*}{ Attribute } & \multirow{2}{*}{ Material } & \multicolumn{3}{|c|}{ Motion Degree Pairs } \\
\hline & & $0-45$ & $0-95$ & 45-95 \\
\hline \multirow{6}{*}{$\begin{array}{l}\tilde{0} \\
\stackrel{0}{0} \\
\tilde{0} \\
0 \\
0\end{array}$} & Glass & 0 & 0 & 0.209 \\
\hline & Metal & 0.269 & 0.003 & 0.178 \\
\hline & Paint & 0.017 & 0 & 0.373 \\
\hline & Plastic & 0.004 & 0.800 & 0.892 \\
\hline & Rubber & 0.070 & 0.003 & 0.553 \\
\hline & Stone & 0.002 & 0 & 0.665 \\
\hline \multirow{6}{*}{ 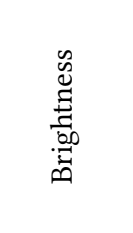 } & Glass & 0 & 0 & 0.988 \\
\hline & Metal & 0.019 & 0 & 0.649 \\
\hline & Paint & 0 & 0 & 0.645 \\
\hline & Plastic & 0.002 & 0 & 0.380 \\
\hline & Rubber & 0.005 & 0 & 0.783 \\
\hline & Stone & 0 & 0 & 0.946 \\
\hline \multirow{6}{*}{ 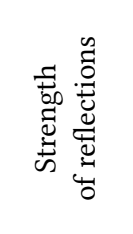 } & Glass & 0.006 & 0 & 0.357 \\
\hline & Metal & 0.014 & 0.004 & 0.108 \\
\hline & Paint & 0.029 & 0.012 & 0.946 \\
\hline & Plastic & 0.003 & 0.005 & 0.274 \\
\hline & Rubber & 0.056 & 0.040 & 0.991 \\
\hline & Stone & 0.003 & 0 & 0.768 \\
\hline \multirow{6}{*}{ 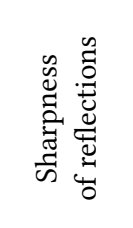 } & Glass & 0 & 0 & 0.592 \\
\hline & Metal & 0.003 & 0.002 & 0.192 \\
\hline & Paint & 0.002 & 0.071 & 0.703 \\
\hline & Plastic & 0 & 0.008 & 0.729 \\
\hline & Rubber & 0.006 & 0 & 0.728 \\
\hline & Stone & 0.027 & 0.004 & 0.918 \\
\hline
\end{tabular}

significantly change under motion, we further investigate the behavior of our perceived brightness. In the following section, we explain the steps carried on in order to understand the effect of motion on our perception of brightness.

\subsection{Influence of brightness}

To further investigate the influence of motion on perceived brightness, we run new experiments using rubber and plastic materials, including additional motion degrees, and adding more levels of luminance in the stimuli.

Stimuli. We generate a series of stimuliu for the materials rubber and plastic, with ten continuous-changed luminance levels (1 to 10) under seven different motion degrees $(0,5,15,45,75,95,135)$. The stimuli used in this experiment for the material rubber can be seen in Figure 1, c). All the stimuli are shown in the supplementary material.

Participants. Similar to the previous user-study, we relied on Amazon Mechanical Turk to launch the experiment. A total of 210 
Matte

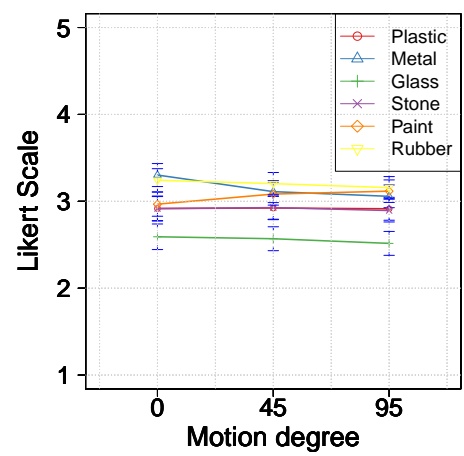

Roughness

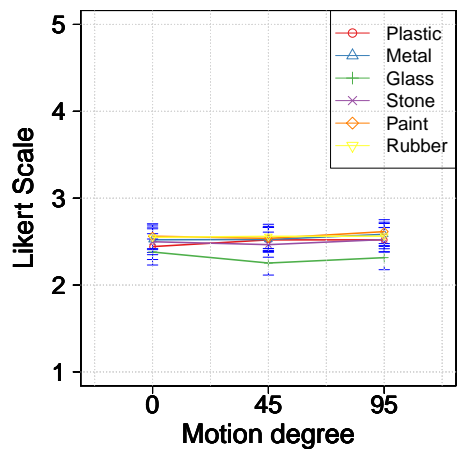

Glossiness

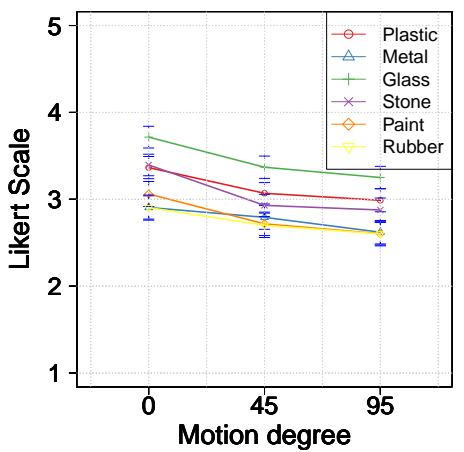

Strength

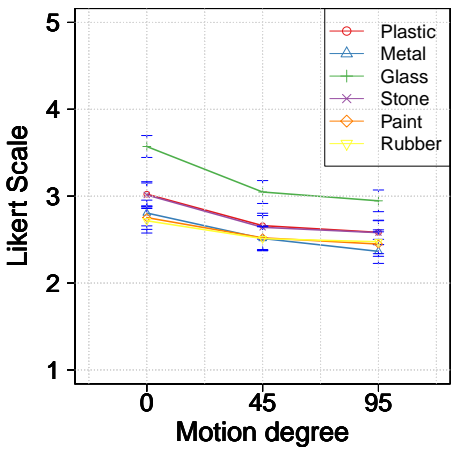

Brightness

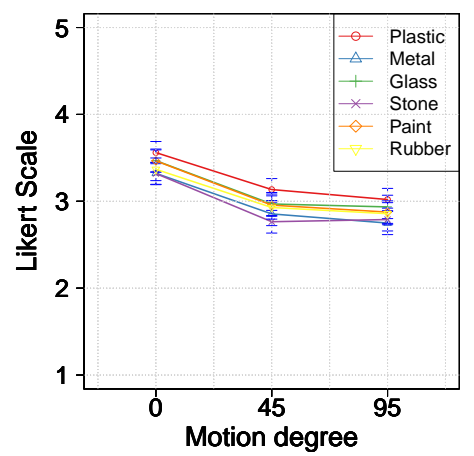

Sharpness

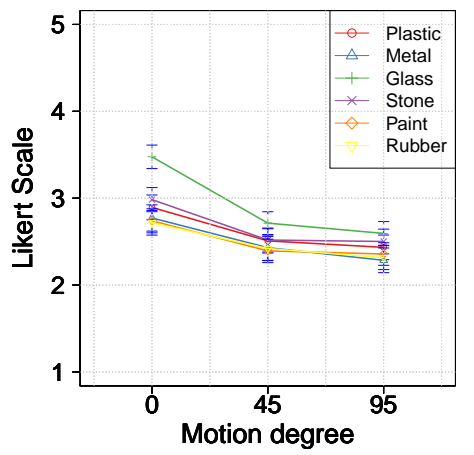

Figure 4: Plot showing the average rating given in the user-study for each of the six perceptual attributes analyzed, for each material type and for all the motion degrees. The $x$-axis shows the variation in motion of the stimuli, the $y$-axis displays the average rating given by the participants. Each color of the line plot represents one of the material categories. Error bars correspond to a 95\% confidence interval. We see how for the attributes glossines, brightness, sharpness of reflections and strength of reflections, there is clear decreasing trend while matte and roughness shows an almost uniform behavior. All the plots for each attribute can be found in the supplementary material.

unique workers took part in it. Users were unaware of the purpose of the experiment.

Procedure. This experiment follows a similar scheme to the one explained in Section 4. Participants are asked to rate a subset of six different attributes (introduced in Section 4) on a continuous 5-point Likert-like scale. Also, although we are only interested in brightness, in order to avoid participants to know the real purpose of the experiment, they are asked to rate all the subset of 6 attributes. We reduce the number of attributes to rate from 14 to 6 in order to avoid distractions in the user [?]. Each experiment has 10 different trials containing all the brightness levels and random motion degrees. The trials are presented randomly to the participants. We collect 15 answers for each sphere, brightness, and motion degree. In the end, we gather a total of 2100 answers.

\subsection{Analysis}

After collecting all the data, we have a set of brightness' ratings for stimuli with variations in their motion degree and luminance. With it, we can build two-dimensional maps that tell us the average perceived brightness for each luminance and motion degree level.
Brightness map construction. In Figure 5, we can see the brightness maps for the materials rubber (left) and plastic (right) The brightness maps are constructed for all 10 luminance levels, in the $\mathrm{x}$-axis, and 7 motion degrees, in the $y$-axis, of the stimuli. In order to build the brightness map, we get the average perceived brightness values of the participants using the ratings of the experiment. Since we cannot generate an infinite set of stimuli, the points that are not sampled are generated using linear interpolation.

Compensation between luminance and motion degree. Both brightness maps confirm the results from the previous stimuli (Section 4), as motion increases our ratings of perceived brightness decrease. However, when the stimulus reaches a high motion degree our perception of its brightness keeps almost constant. Also, If we follow an isocontour in the brightness maps - the lines that have the same average perceived brightness -, we can observe how, in order to keep a constant perceived brightness, the slope of the isocontour bends. Moreover, if we compare both brightness maps, we can observe how the plastic has an overall less perceived luminance. We argue that this happens due to the blur in the highlights of the plastic, a specular material, produced by the effect of motion 

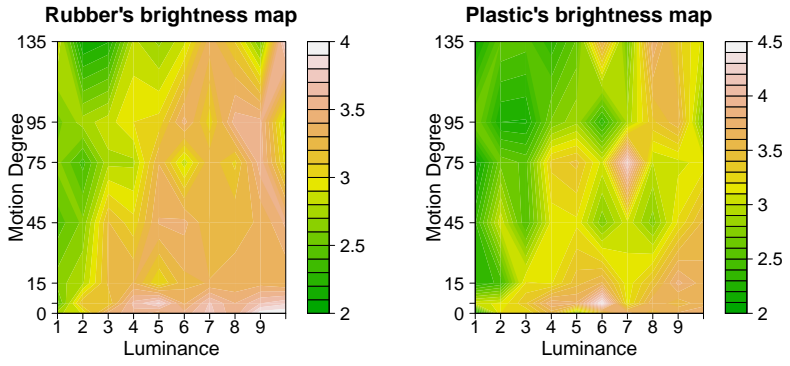

Figure 5: On the left, the rubber brightness map created using the stimuli from the experiments. On the right, the plastic brightness map. For both brightness maps, the $x$-axis is the luminance of the stimulus while the $y$-axis is its motion degree. Both figures show how as motion degree increases, brightness will decrease non-linearly.

blur. While on the rubber, since it is a diffuse material, there are no significant highlights and the effect of motion has a reduced impact compared to the case of plastic.

Luminance region split. Isocontours show how in order to keep the same level of perceived brightness, luminance levels have to increase non-linearly when motion degree is increased linearly. Moreover, if we follow the isocontour generated between the values 2 and 3 of luminance, we can see a clear separation; on the left, we would have the dim area with values of perceived brightness around 2.5 while on the right side of the isocontour we can see a constant area with perceived brightness of 3.5. These two regions are separated by the isocontour where our perceived brightness takes a value of 3 . This exhibits the non-linear behavior of human perception, and also how, although the values of luminance and motion degree are changing, our perception of brightness remains constant.

\section{DISCUSSION AND FUTURE WORK}

\subsection{Conclusion}

We have presented an analysis of the effects of motion blur on our perception of a wide set of material attributes, for different material categories. To do it, we analyze 38,880 ratings $(72$ (materials $) \times$ 3 (motion degrees) $\times 30$ (answers $/$ stimulus $) \times 14$ (attributes)) given for six different material attributes: brightness, glossiness, matte, roughness, sharpness of reflections and strength of reflections. Please refer to the supplemental material for the complete analysis of all 14 attributes included in the experiments.

A reasonable concern when using Mechanical Turk as a source for participants in user studies, is the possible effect of uncontrolled viewing conditions (such as display characteristics or viewing environment). Nevertheless, previous studies have shown that MTurk can actually be used in visual psychophysical experiments, and its results matched those under controlled lab conditions, since a large number of participants reduces variance (e.g. [Jarabo et al. 2014 ; ?]). Similarly, participants were not screened for proficiency in English, so it may be that some subtleties in the description of material appearance were lost.

Our results indicate that motion blur has a significant effect on our perception of attributes related to the specular nature of the material, namely glossiness, brightness, and strength and sharpness of reflections, whereas no significant influence exists on the attributes matte and roughness. The four aforementioned attributesglossiness, brightness, and strength and sharpness of reflections-are given significantly lower ratings as the motion degree increases. This can be due to the removal of high frequencies of the stimuli as a consequence of motion blur, since it has been shown that these relate to specularity [Durand et al. 2005]. When looking at the nature of this influence through post hoc analyses, we observe that, among lower motion degrees the influence of motion blur on the perception of attributes is larger than among higher ones; actually, there is no significant difference between motion degrees 45 and 95, while there is between degrees 0 and 45 . This seems to indicate a stabilization of perceived attribute magnitudes for higher motion degrees, i.e., from a certain motion degree, increased motion blur does not change the perception of these attributes.

Moreover, we select an attribute and material category to do a more in-depth analysis of the influence of motion blur on perceived brightness. In this second experiment, we not only vary the motion degree, but also the luminance of the material shown in the stimuli. We seek to observe how the isocontours (i.e., lines of constant brightness) behave as we vary both motion degree and luminance level. Despite the limited extent of our experiment, we observe how the non-linear behavior with motion degree persists. Further, this behavior is different for different luminance levels, falling back to an almost constant (independent of motion degree) behavior for both very high and very low luminance levels. This intricate relationship suggests the need for more detailed experiments, covering a wider range of appearances and perceived attributes; this work takes the first steps in this direction, but a comprehensive model of the influence of motion blur in material perception remains as future work.

\subsection{Future work}

In this work, we have just investigated the effect of motion blur due to linear motion in our perception of material appearance; other kinds of motion, including rotation, multidirectional paths, accelerated movement, etc., would require further analysis, for which we hope our work can provide a solid basis. Similarly, we make the reasonable assumption that motion blurred images are a good proxy for actual moving stimuli for the purposes of our test.

Our stimuli have been rendered using homogeneous material files in MDL. Exploring how the missing information encoded in the heterogeneities of some materials (like stone, or wood) affects appearance perception is an interesting extension, not included in our work. Some materials, especially specular ones, may require a wider dynamic range than what a typical display provides, an aspect of appearance perception not covered in this work since our stimuli were tone mapped. Also, since users, in general, tend to avoid extrema in Likert-based tests, it would be interesting to find if using a 7-point scale would have an influence in their responses. Finally, extending this work to find a correlation between image 
statistics and the set of perceptual attributes analyzed remains unexplored. Human perception of appearance is a sophisticated process, not fully understood, which poses many challenges and opens interesting avenues of future research, and we hope our work will inspire future exploration of the influence of motion in our perception. For instance, our findings could be used to optimize the performance of rendering pipelines where motion can have a big influence like in the emerging fields of virtual reality or real-time ray-tracing.

\section{ACKNOWLEDGMENTS}

We want to thank the anonymous reviewers for their encouraging and insightful feedback on the manuscript; the members of the Graphics \& Imaging Lab for fruitful discussion, and in particular Ana Serrano Pacheu for the website building, Ibón Guillen, Sandra Malpica, Julio Marco, Marta Ortín Obón and Incheol Kim for help on the pilot experiments. Ruiquan Mao also would like to thank Davide Deflorio, PhD candidate in SyMoN Lab, University of Birmingham, for the valuable suggestions. Manuel Lagunas is funded by European Research Council (ERC) under the European Union's Horizon 2020 research and innovation programme (CHAMELEON project, grant agreement No 682080). Ruiquan Mao is funded by Marie Skłodowska-Curie Action (MSCA-ITN/ETN) under the European Union's Horizon 2020 research and innovation programme (DyViTo project, grant agreement No 765121).

\section{REFERENCES}

Carlos Aliaga, Carol O’Sullivan, Diego Gutierrez, and Rasmus Tamstorf. 2015a. Sackcloth or silk?: the impact of appearance vs dynamics on the perception of animated cloth. In Proceedings of the ACM SIGGRAPH symposium on applied perception. ACM, 41-46.

Carlos Aliaga, Carol O'Sullivan, Diego Gutierrez, and Rasmus Tamstorf. 2015b. Sackcloth or silk?: the impact of appearance vs dynamics on the perception of animated cloth. In Proceedings of the ACM SIGGRAPH symposium on applied perception. ACM, 41-46.

Barton L Anderson. 2011. Visual perception of materials and surfaces. Current Biology 21, 24 (2011), R978-R983.

G. Bradski. 2000. The OpenCV Library. Dr. Dobb's fournal of Software Tools (2000)

Myron L Braunstein. 2014. Depth perception through motion. Academic Press.

Rebecca A Champion and Paul A Warren. 2017. Contrast effects on speed perception for linear and radial motion. Vision research 140 (2017), 66-72.

Katja Doerschner, Roland W. Fleming, Ozgur Yilmaz, Paul R. Schrater, Bruce Hartung, and Daniel Kersten. 2011. Visual Motion and the Perception of Surface Material. Current Biology 21, 23 (2011), 2010 - 2016.

Song-Pei Du, Belen Masia, Shi-Min Hu, and Diego Gutierrez. 2013. A metric of visual comfort for stereoscopic motion. ACM Transactions on Graphics (TOG) 32, 6 (2013), 222.

Jonathan Dupuy and Wenzel Jakob. 2018. An Adaptive Parameterization for Efficient Material Acquisition and Rendering. ACM Trans. Graph.

Frédo Durand, Nicolas Holzschuch, Cyril Soler, Eric Chan, and François X Sillion. 2005. A frequency analysis of light transport. ACM Transactions on Graphics (TOG) 24, 3 (2005), 1115-1126

Jirí Filip and Radomír Vávra. 2014. Template-based sampling of anisotropic BRDFs. In Computer Graphics Forum, Vol. 33. Wiley Online Library, 91-99.

Roland W. Fleming. 2014. Visual perception of materials and their properties. Vision Research 94 (2014), 62 - 75.

Roland W Fleming and Heinrich H Bülthoff. 2005. Low-level image cues in the perception of translucent materials. ACM Transactions on Applied Perception (TAP) 2, 3 (2005), 346-382.

Roland W Fleming, Ron O Dror, and Edward H Adelson. 2003. Real-world illumination and the perception of surface reflectance properties. Fournal of vision 3, 5 (2003), 3-3.

Roland W Fleming, Karl R Gegenfurtner, and Shin'ya Nishida. 2015a. Visual perception of materials: the science of stuff. Vision research 109 (2015), 123-124.

Roland W. Fleming, Shin'ya Nishida, and Karl R. Gegenfurtner. 2015b. Perception of material properties. Vision Research 115 (2015), 157 - 162.
Roland W Fleming, Christiane Wiebel, and Karl Gegenfurtner. 2013. Perceptual qualities and material classes. Fournal of vision 13, 8 (2013), 9-9.

Adria Fores, James Ferwerda, and Jinwei Gu. 2012. Toward a perceptually based metric for BRDF modeling. In Color and Imaging Conference, Vol. 2012. Society for Imaging Science and Technology, 142-148.

Elena Garces, Aseem Agarwala, Diego Gutierrez, and Aaron Hertzmann. 2014. A Similarity Measure for Illustration Style. ACM Transactions on Graphics (SIGGRAPH 2014) 33,4 (2014)

Guillaume Ged, Gaël Obein, Zaccaria Silvestri, Jean Le Rohellec, and Françoise Viénot. 2010. Recognizing real materials from their glossy appearance. fournal of vision 10 , 9 (2010), 18-18.

Ioannis Gkioulekas, Bruce Walter, Edward H Adelson, Kavita Bala, and Todd Zickler. 2015. On the appearance of translucent edges. In Proceedings of the IEEE Conference on Computer Vision and Pattern Recognition. 5528-5536.

Bruce Hartung and Daniel Kersten. 2002. Distinguishing shiny from matte. Journal of Vision 2, 7 (2002), 551-551.

Vlastimil Havran, Jiri Filip, and Karol Myszkowski. 2016. Perceptually Motivated BRDF Comparison using Single Image. Computer Graphics Forum (2016).

Adrian Jarabo, Hongzhi Wu, Julie Dorsey, Holly Rushmeier, and Diego Gutierrez. 2014. Effects of Approximate Filtering on the Appearance of Bidirectional Texture Functions. IEEE Trans. on Visualization and Computer Graphics 20, 6 (2014).

Tae-Eui Kam, Damien J Mannion, Seong-Whan Lee, Katja Doerschner, and Daniel J Kersten. 2015. Human visual cortical responses to specular and matte motion flows. Frontiers in human neuroscience 9 (2015), 579.

Takahiro Kawabe, Kazushi Maruya, Roland W Fleming, and Shin'ya Nishida. 2015. Seeing liquids from visual motion. Vision research 109 (2015), 125-138.

L. Kettner, M. Raab, D. Seibert, J. Jordan, and A. Keller. 2015. The Material Definition Language. In Workshop on Material Appearance Modeling, Reinhard Klein and Holly Rushmeier (Eds.). The Eurographics Association. https://doi.org/10.2312/mam. 20151195

Jaroslav Křivánek, James A. Ferwerda, and Kavita Bala. 2010. Effects of global illumination approximations on material appearance. ACM Trans. Graph. 29, 4, Article 112 (July 2010), 112:1-112:10 pages. SIGGRAPH '10.

Manuel Lagunas, Elena Garces, and Diego Gutierrez. 2018. Learning icons appearance similarity. Multimedia Tools and Applications (2018), 1-19.

Manuel Lagunas, Sandra Malpica, Ana Serrano, Elena Garces, Diego Gutierrez, and Belen Masia. 2019. A Similarity Measure for Material Appearance. ACM Transactions on Graphics (SIGGRAPH 2019) 38, 4 (2019).

Wenbin Li and Mario Fritz. 2012. Recognizing materials from virtual examples. In European Conference on Computer Vision. Springer, 345-358.

Stephen Lombardi and Ko Nishino. 2012. Reflectance and natural illumination from a single image. In European Conference on Computer Vision. Springer, 582-595.

Laurence T Maloney and David H Brainard. 2010. Color and material perception: Achievements and challenges. Fournal of Vision 10, 9 (2010), 19-19.

Phillip J Marlow and Barton L Anderson. 2016. Motion and texture shape cues modulate perceived material properties. fournal of vision 16, 1 (2016), 5-5.

Wojciech Matusik, Hanspeter Pfister, Matt Brand, and Leonard McMillan. 2003. A Data-Driven Reflectance Model. ACM Transactions on Graphics 22, 3 (July 2003), 759-769.

Takehiro Nagai, Toshiki Matsushima, Kowa Koida, Yusuke Tani, Michiteru Kitazaki, and Shigeki Nakauchi. 2015. Temporal properties of material categorization and material rating: visual vs non-visual material features. Vision Research 115 (2015), 259 - 270. https://doi.org/10.1016/j.visres.2014.12.011 Perception of Material Properties (Part II).

Fernando Navarro, Susana Castillo, Francisco J. Serón, and Diego Gutierrez. 2011. Perceptual considerations for motion blur rendering. ACM Transactions on Applied Perception 8, 3 (2011), 1-15. https://doi.org/10.1145/2010325.2010330 arXiv: 1710.03346

HAROLD T NEFS. 2008. On the visual appearance of objects. In Product experience. Elsevier, 11-39.

Addy Ngan, Frédo Durand, and Wojciech Matusik. 2005. Experimental Analysis of BRDF Models. In Eurographics Symposium on Rendering (2005). The Eurographics Association.

Addy Ngan, Frédo Durand, and Wojciech Matusik. 2006. Image-driven Navigation of Analytical BRDF Models.. In Rendering Techniques. 399-407.

Jannik Boll Nielsen, Henrik Wann Jensen, Ravi Ramamoorthi, and San Diego. 2015. On Optimal , Minimal BRDF Sampling for Reflectance Acquisition. ACM Transactions on Graphics 34, 6 (2015), 1-11. https://doi.org/10.1145/2816795.2818085

Steven G Parker, James Bigler, Andreas Dietrich, Heiko Friedrich, Jared Hoberock, David Luebke, David McAllister, Morgan McGuire, Keith Morley, Austin Robison, et al. 2010. OptiX: a general purpose ray tracing engine. In Acm transactions on graphics (tog), Vol. 29. ACM, 66.

Fabio Pellacini, James A. Ferwerda, and Donald P. Greenberg. 2000. Toward a Psychophysically-based Light Reflection Model for Image Synthesis. In Proceedings of the 27th Annual Conference on Computer Graphics and Interactive Techniques (SIGGRAPH '00). 55-64.

Thiago Pereira and Szymon Rusinkiewicz. 2012. Gamut mapping spatially varying reflectance with an improved BRDF similarity metric. In Computer Graphics Forum, Vol. 31. Wiley Online Library, 1557-1566. 
T Pohlert. 2018. PMCMRplus: calculate pairwise multiple comparisons of mean rank sums extended. R package version 1, 0 (2018).

Michael Rubinstein, Diego Gutierrez, Olga Sorkine, and Ariel Shamir. 2010. A Comparative Study of Image Retargeting. ACM Transactions on Graphics (Proc. SIGGRAPH Asia) 29, 6 (2010), 160:1-160:10

Y Sakano. 2008. Effects of self-motion on gloss perception. Perception 37 ECVP Abstract Supplement, 200877 (2008)

Ana Serrano, Diego Gutierrez, Karol Myszkowski, Hans-Peter Seidel, and Belen Masia 2016. An Intuitive Control Space for Material Appearance. ACM Trans. Graph. 35, 6, Article 186 (Nov. 2016), 186:1-186:12 pages.

Lavanya Sharan, Ruth Rosenholtz, and Edward Adelson. 2009. Material perception What can you see in a brief glance? Journal of Vision 9, 8 (2009), 784-784.

Lavanya Sharan, Ruth Rosenholtz, and Edward H Adelson. 2008. Eye movements for shape and material perception. Fournal of Vision 8, 6 (2008), 219-219.

Cyril Soler, Kartic Subr, and Derek Nowrouzezahrai. 2018. A Versatile Parameterization for Measured Material Manifolds. In Computer Graphics Forum, Vol. 37. Wiley Online Library, 135-144.

Tiancheng Sun, Ana Serrano, Diego Gutierrez, and Belen Masia. 2017. Attributepreserving Gamut Mapping of Measured BRDFs. Comput. Graph. Forum 36, 4 (July 2017).

Yusuke Tani, Keisuke Araki, Takehiro Nagai, Kowa Koida, Shigeki Nakauchi, and Michiteru Kitazaki. 2013. Enhancement of glossiness perception by retinal-image motion: Additional effect of head-yoked motion parallax. PloS one 8, 1 (2013), e54549.
William Thompson, Roland Fleming, Sarah Creem-Regehr, and Jeanine Kelly Stefanucci. 2011. Visual Perception from a Computer Graphics Perspective (1st ed.). A. K. Peters, Ltd., Natick, MA, USA.

Shimon Ullman. 1979. The interpretation of visual motion. Massachusetts Inst of Technology Pr.

Peter Vangorp, Jurgen Laurijssen, and Philip Dutré. 2007. The Influence of Shape on the Perception of Material Reflectance. ACM Transactions on Graphics 26, 3, Article 77 (July 2007).

Peter Welinder, Steve Branson, Pietro Perona, and Serge J Belongie. 2010. The multidimensional wisdom of crowds. In Advances in neural information processing systems. $2424-2432$.

Josh Wills, Sameer Agarwal, David Kriegman, and Serge Belongie. 2009. Toward a Perceptual Space for Gloss. ACM Trans. Graph. 28, 4, Article 103 (Sept. 2009), 103:1-103:15 pages.

Mehmet Ersin Yumer, Siddhartha Chaudhuri, Jessica K Hodgins, and Levent Burak Kara. 2015. Semantic shape editing using deformation handles. ACM Transactions on Graphics (TOG) 34, 4 (2015), 86.

Johannes M Zanker. 1995. Does motion perception follow Weber's law? Perception 24, 4 (1995), 363-372.

Eduard Zell, Carlos Aliaga, Adrian Jarabo, Katja Zibrek, Diego Gutierrez, Rachel McDonnell, and Mario Botsch. 2015. To stylize or not to stylize?: the effect of shape and material stylization on the perception of computer-generated faces. $A C M$ Transactions on Graphics (TOG) 34, 6 (2015), 184

Károly Zsolnai-Fehér, Peter Wonka, and Michael Wimmer. 2018. Gaussian Material Synthesis. ACM Trans. Graph. 37, 4, Article 76 (July 2018), 76:1-76:14 pages. 